\title{
Médiévales
}

Langues, Textes, Histoire

70 | printemps 2016

Lieux d'hygiène et lieux d'aisance en terre d'Islam (VII $\mathrm{XV}^{\mathrm{e}}$ siècle)

\section{Les latrines dans l'architecture domestique et palatiale de Palerme, un marqueur social ?}

Latrines in the Domestic and Palatial Architecture of Palermo

\section{Elena Pezzini}

Traducteur : Laurence Moulinier-Brogi

\section{(2) OpenEdition}

\section{Journals}

Édition électronique

URL : http://journals.openedition.org/medievales/7839

DOI : 10.4000/medievales.7839

ISSN : 1777-5892

\section{Éditeur}

Presses universitaires de Vincennes

\section{Édition imprimée}

Date de publication : 23 juin 2016

Pagination : 171-188

ISSN : 0751-2708

\section{Référence électronique}

Elena Pezzini, «Les latrines dans l'architecture domestique et palatiale de Palerme, un marqueur social ? », Médiévales [En ligne], 70 I printemps 2016, mis en ligne le 15 juin 2018, consulté le 22 avri 2019. URL : http://journals.openedition.org/medievales/7839 ; DOI : 10.4000/medievales.7839 
Elena Pezzini

\section{Les latrines dans l'architecture domestique et palatiale de Palerme, un marqueur social ?}

En 1959, Leopoldo Torres Balbás, dans un article sur les latrines ${ }^{1}$ d'al-Andalus, affirmait que la perfection du système adopté pour approvisionner en eau et pour résoudre le problème des eaux usées renvoyait l'image d'une civilisation urbaine raffinée davantage que « la richesse, la grandeur ou même la perfection des édifices qu'elle avait érigés ${ }^{2}$ ».

Dans le cas de la Palerme musulmane, la possibilité de mesurer la qualité de la vie urbaine à l'aune des latrines se heurte à la rareté de la documentation : on ne dispose en effet que de peu de sources permettant de déterminer s'il y en avait, et - si oui - quelles étaient les caractéristiques de ces lieux destinés à l'hygiène. La documentation les concernant, à Palerme et dans les autres centres urbains de Sicile, est essentiellement tardive. On est bien loin de la richesse des attestations qui a permis à Ieva Reklaityte d'affirmer que la majeure partie des maisons d'al-Andalus en disposait, et d'analyser les solutions adoptées pour éliminer leurs rejets ${ }^{3}$. Les recherches archéologiques menées à Palerme n'ont mis au jour aucune latrine, si l'on en juge par les résultats publiés ; toutefois, cette donnée prend un poids relatif si l'on considère que ces mêmes enquêtes ne permettent pas de reconstruire intégralement ne fût-ce qu'une structure d'habitation.

Cette pauvreté de la documentation archéologique est imputable à deux faits : avant tout, la cité moderne s'est superposée à la ville médiévale en réutilisant les espaces, mais aussi les matériaux, avec des activités de

1. On entend ici par latrine une pièce spécialement aménagée pour l'accomplissement des besoins naturels et pourvue d'installations d'évacuation idoines.

2. L. TORRES BALBÁs, « Letrinas y bacines », Al-Andalus, 24.1 (1959), p. 221-234 (p. 221).

3. I. ReKLAITYTE, «El saneamiento en la ciudade andalusíes », Anales de Arqueología Cordobesa, 16 (2005), p. 207-238. 
spoliation amplement documentées ${ }^{4}$; ensuite, l'intérêt des archéologues pour la ville médiévale ne s'est développé qu'à partir de la fin des années $1980^{5}$, et ce n'est que récemment, grâce à un intérêt renouvelé porté par Annliese Nef et Alessandra Bagnera ${ }^{6}$, que la ville islamique, invisible il y a quelques années encore, ou lisible seulement à travers les sources écrites ${ }^{7}$, commence lentement à sortir de l'ombre ${ }^{8}$. Plus que tout, la révision des indicateurs chronologiques impulsée par Alessandra Bagnera ${ }^{9}$ a permis de poser de nouvelles questions, et de donner une épaisseur historique à une période de la vie urbaine qui apparaissait comprimée dans une dimension anhistorique.

Toutefois, si certains aspects de la culture matérielle et de la céramique en particulier commencent à être plus clairs, les données relatives à l'architecture monumentale en général font défaut ${ }^{10}$, tandis que celles sur l'architecture domestique sont très lacunaires, et substantiellement limitées à des vestiges d'habitat récemment mis au jour lors de travaux au Palazzo

4. La spoliation est attestée, par exemple, par le fait que les structures normandes sont construites avec des pierres et des tuiles de réemploi ; cf. G. SPATRISANO, La Zisa e lo Scibene di Palermo, Palerme, 1982, p. 32.

5. Après les recherches menées lors des chantiers de restauration du Steri et de la Zisa au début des années 1970, un premier programme d'archéologie urbaine a été lancé au milieu des années 1980, avec les fouilles réalisées à Rione Castello San Pietro par la Soprintendenza per i Beni Culturali e Ambientali di Palermo et par l'École Française de Rome.

6. L. ArCiFA, A. BAGNERA et A. NeF, «Archeologia della Sicilia islamica : nuove proposte di riflessione », dans P. SÉNAC éd., Histoire et archéologie de l'Occident musulman (VII $-X V^{e}$ siècle), Al-Andalus, Maghreb, Sicile, Villa 4, Toulouse, 2012, p. 241-274 ; A. BAGNERA, « From a Small Town to a Capital : The Urban Evolution of Islamic Palermo (9th-mid-11th Century) », dans A. NEF éd., A Companion to Medieval Palermo. The History of a Mediterranean City from 600 to 1500, Leyde/Boston 2013, p. 61-88 ; A. NEF, «Islamic Palermo and the Dār al-Islām : Politics, Society and Economy (from the mid-9th to the mid11th Century) », dans EAD. éd., A Companion..., p. 39-59 ; A. BAGNERA et A. NEF, « Histoire et archéologie du domaine fatimide : la Sicile et Palerme (début du $\mathrm{X}^{\mathrm{e}}$-milieu du XI $\mathrm{XI}^{\mathrm{e}}$ siècle) », dans P. Cressier et M. RAmmah éd., en préparation ; A. NeF, "Croissance urbaine et modalités d'habitat dans la Palerme islamique : retour sur les acteurs et les facteurs », dans L. ARCifa et M. R. Sgarlata éd., From polis to Medina. La trasformazione delle città siciliane tra tardo antico e alto medioevo (Siracuse, 21-23 giugno 2012), sous presse.

7. Voir les remarques d'A. De Simone, dans son article fondamental, A. DE SIMONE, « Palermo araba. Custodia Panormi est : intra baych et ferach...», dans R. LA DuCA éd., Storia di Palermo II. Dal tardo-antico all'Islam, Palerme, 2000, p. 78-113.

8. Voir les textes consacrés à la Palerme islamique dans F. ARDIZZONE et A. NEF éd., Les Dynamiques de l'islamisation en Méditerranée centrale et en Sicile : nouvelles propositions et découvertes récentes (Palerme, 8-10 novembre 2012), Rome/Bari, 2014.

9. L. ARCIFA, A. BAGNERA et A. NEF, «Archeologia della Sicilia islamica... », p. 245256 ; A. BAGNERA, « La ceramica invetriata di età islamica », dans EAD. éd., Archeologia dell'Islam in Sicilia, catalogo della mostra, Gibellina, 2012.

10. C'est très récemment qu'a été identifiée une phase islamique bien reconnaissable sous la construction monumentale du palais de Maredolce (S. VASSALlO et E. CANZONIERI, "Insediamenti extraurbani a Palermo : nuovi dati da Maredolce », dans A. NEF et F. ARdizzone éd., Les Dynamiques..., p. 271-280). 
Statella Spaccaforno ${ }^{11}$. D'autres recherches n'ont restitué que de nouvelles traces d'habitat répondant à un urbanisme planifié, se caractérisant par la régularité de l'implantation et la densité du tissu ${ }^{12}$.

\section{Habitat et latrines à l'époque islamique}

Les sources écrites brossent de Palerme (fig. 1) le portrait d'une ville à la trame non homogène, et des travaux récents ont émis l'hypothèse selon laquelle une architecture résidentielle de prestige se serait concentrée dans la zone correspondant au noyau antique, entouré de murailles, l'antique Panormos, devenue par suite Madīnat Balarm ou Qașr al-Qadīm ${ }^{13}$. Les sources sur la conquête de la ville par les Normands localisent dans cette zone les vieilles demeures des «Sarrasins », qui étaient vraisemblablement des palais, et dont pour l'heure il semble qu'on n'ait gardé aucune trace. Dans cette partie de la ville islamique, bordée par deux cours d'eau, il est possible qu'aient existé des systèmes d'écoulement vers ceux-ci ; la décharge des eaux dans les voies secondaires est attestée à l'époque romaine $^{14}$; pour l'époque tardo-médiévale, des conduites reliées aux latrines ont été documentées ${ }^{15}$, alors qu'il n'existe pas de réseau public d'assainissement. Malheureusement, puisque le maintien d'une bonne partie de la trame viaire est attesté depuis l'époque hellénistique jusqu'à nos jours ${ }^{16}$, les systèmes d'égouts et les équipements récents ont interdit de

11. F. SPATAFORA et E. CANZONIERI, «Al-Khālișa : alcune considerazioni alla luce delle nuove scoperte archeologiche nel quartiere della Kalsa », dans A. NEF et F. ARDIZZONE éd., Les Dynamiques..., p. 233-245.

12. A. BAGNERA, « From a Small Town... », p. 82-83, avec bibliographie.

13. E. PEZZINI, «Palermo in the 12th Century. Transformations in forma urbis », dans A. NeF éd., A Companion..., p. 221-222 ; A. NEF, « Croissance urbaine et modalités d'habitat... ».

14. F. Spatafora, M. Montali, « Palermo : nuovi scavi nell'area di piazza della Vittoria », dans M. OSANNA, M. TORELLI, Sicilia ellenistica, consuetudo italica. Alle origini dell'architettura ellenistica d'Occidente, Rome, 2006, p. 133-151 (p. 135-136). Les maisons romaines de piazza della Vittoria présentent des canalisations qui présupposent aussi des systèmes d'évacuation des eaux.

15. Un acte de la première moitié du XIV ${ }^{\mathrm{e}}$ siècle atteste de la vente d'un catoio (catogium, pièce basse) libre de toute servitude, sauf pour une mater latrine se trouvant dans le sol du catoio et qui devait y rester encore dix ans, au terme desquels les vendeurs s'engagent à la transférer dans un autre lieu (Palermo, Archivio di Stato di Palermo, Spezzoni Notarili, 327N, c. 1r, ante 1348). Le catoio jouxte la maison des vendeurs et, en particulier, sur un côté, la sikifa de la maison. Le terme de sikifa, dérivé de l'arabe, est utilisé pour indiquer un type particulier de vestibule, et donne à penser que la maison des vendeurs était une des maisons anciennes du quartier. La mater latrine était probablement un système d'élimination des eaux usées, ou des déchets de la latrine de la domus qui se trouvait au-dessus. Quoi qu'il en soit, le contexte indique que la mater latrine est privée.

16. F. Spatafora et M. Montali, « Palermo : nuovi scavi... », p. 134. 


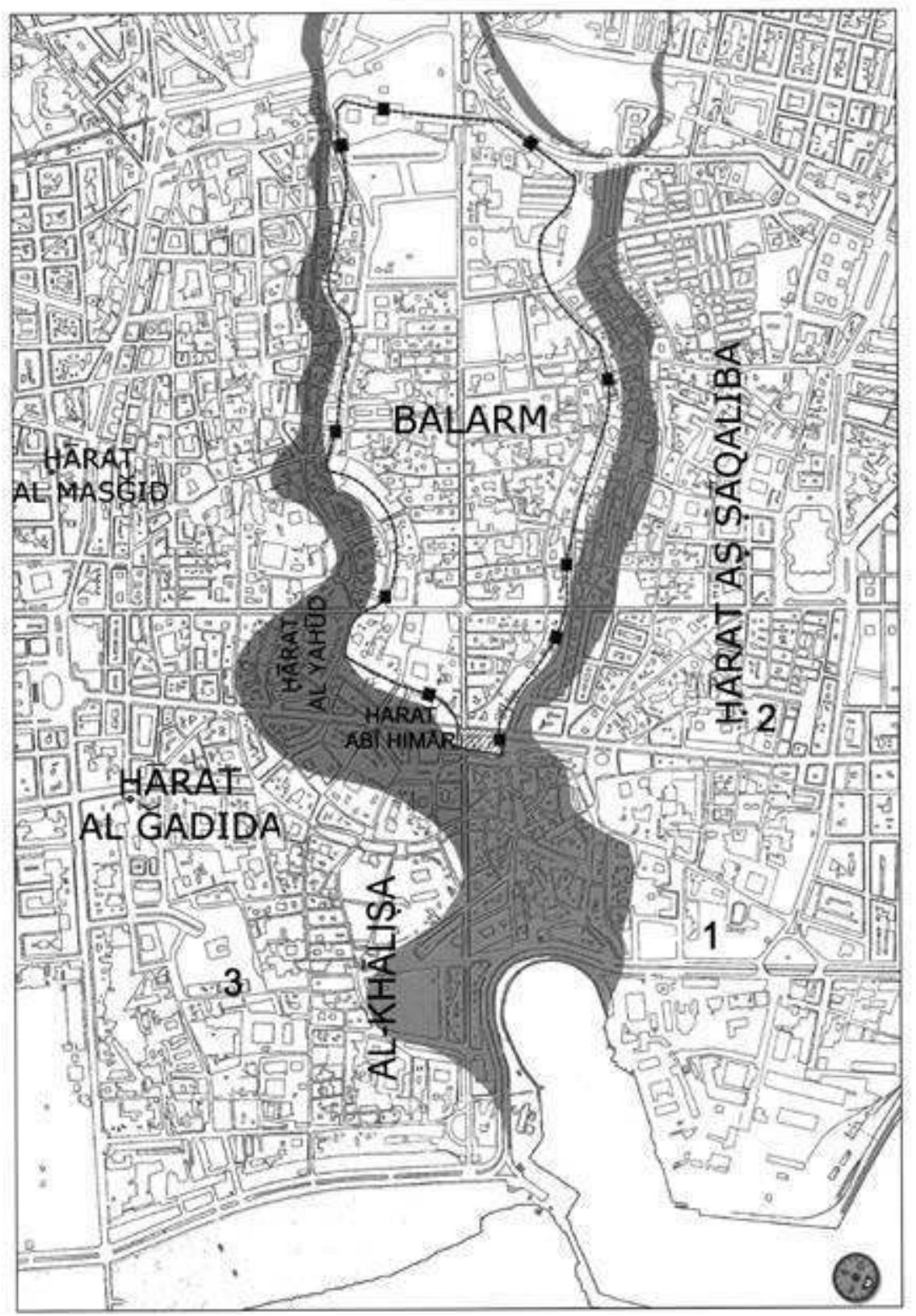

Fig. 1. Palerme islamique

Balarm : correspondant au noyau antique (Panormos).

Ḥārat al-Șaqāliba, al-Khāliṣa, Hāarat al-Djadīda, Hāarat al-Masdjid : développés à l'époque islamique . Recherches archéologiques citées dans le texte : 1. Castello San Pietro ; 2. Ancien couvent des Pères Philippins all'Olivella, aujourd'hui siège du Musée Archéologique Régional « Antonino Salinas »; 3. Palais Statella Spaccaforno.

(Graphiques par Maria Antonietta Parlapieno et Margherita Accascina) 
vérifier si des infrastructures plus anciennes avaient existé, en particulier celles liées aux axes antiques principaux.

D'autre part, les recherches récentes menées dans les quartiers s'étant développés à l'époque islamique en dehors du noyau emmuré antique ont produit des données contradictoires et d'interprétation difficile. Bien que l'état de conservation des structures d'habitation n'ait pas permis, pour le moment, d'identifier des espaces destinés aux latrines, on peut penser cependant que certaines des nombreuses fosses localisées dans différentes zones urbaines et colmatées par des déchets, interprétées tour à tour comme des carrières ou des silos ${ }^{17}$, pourraient avoir eu plutôt une fonction de puits noirs et être donc les seuls vestiges de latrines situées au-dessus.

L'existence de latrines dans la Sicile islamique ne fait pas de doute. Un document relatif à la ville de Catane l'atteste : dans la Djarīda de 1145, qui renouvelle à l'église de Catane la platea que le comte Roger lui avait assignée cinquante ans plus tôt, apparaît l'anthroponyme al-kannāf, c'està-dire « vide-latrines » ou vidangeur ${ }^{18}$. Comme l'anthroponyme est arabe et que la liste remonte à la fin du $\mathrm{XI}^{\mathrm{e}}$ siècle, l'indice semble fort. Il est probable, de ce point de vue, que la réalité palermitaine n'ait pas été différente de celle de Catane et que la présence de vidangeurs suppose l'existence de latrines et de leurs puits perdus.

En effet à Palerme, des fosses auraient été découvertes dans le vaste quartier identifié avec le Hāarat al-Ṣaqāliba. Les recherches menées dans diverses zones de celui-ci ont mis au jour une documentation archéologique extrêmement fragmentaire, mais qui permet de reconnaître les résultats de plusieurs phases de planification urbaine ${ }^{19}$.

Dans le quartier Castello San Pietro, un puits à embouchure ovale s'élargissant vers le fond selon un profil en cloche a été documenté à la base d'un mur, à l'intérieur de la pièce 5 . La fouille a mis en évidence des opérations successives de curage du puits, qui présentait sur ses parois des restes de concrétions noires, et dans le fond une série de couches verdâtres et noires. L'installation du puits est liée à la première phase d'une urbanisation planifiée de la zone, datable d'avant le milieu du $\mathrm{X}^{\mathrm{e}}$ siècle ; son oblitération

17. P. TODARO, Il sottosuolo di Palermo, Palerme, 1988.

18. A. DE SIMONE, Spoglio antroponimico delle giaride arabo-greche dei diplomi editi da Salvatore Cusa, Rome, 1979, p. 24, 62. Sur cette donnée et sur la possibilité de la généraliser à Palerme où les Consuetudines urbaines, formalisées au XIII ${ }^{\mathrm{e}}$ siècle, indiquent des aires hors la ville destinées à la décharge des déchets, voir E. PEZZINI, « Lo smaltimento dei rifiuti a Palermo nel Bassomedioevo : dati documentari », dans M. MILANESE, V. CAMINNECI, M. C. PARello, M. S. Rizzo éd., Dal butto alla storia. Indagini archeologiche tra Medioevo e Postmedioevo, Atti del Convegno (Sciacca/Burgio/Ribera 28-29 mars 2011), Archeologia Postmedievale, 16 (2012), p. 13-22 (p 18, 20).

19. A. BAGNERA, « From a Small Town... », p. 82. 
a eu lieu à l'époque tardo-islamique, avec un remplissage effectué en une seule fois, se substituant aux strates sombres dont il ne reste que des traces.

Il pourrait donc s'agir d'un puisard relié à une latrine, vidé régulièrement de son contenu, et qui, une fois devenu obsolète, fut comblé avec des apports secondaires. Dans le cadre de ces mêmes recherches a été retrouvé, le long d'un axe de rue, un segment de canalisation qui servait peut-être à l'écoulement des eaux de pluie ${ }^{20}$.

Les sondages réalisés dans les sous-sols du couvent des Pères Philippins all'Olivella, aujourd'hui siège du Musée archéologique régional «Antonino Salinas », ont révélé un réseau de puits et de cavités en forme d'entonnoirs qui confirment l'urbanisation intensive de la zone ; toutefois, ces traces sont extrêmement ténues ${ }^{21}$ en raison des destructions liées à l'édification du couvent. Ces cavités ont été identifiées comme des silos ou parfois comme des carrières, étant donné que leurs parois sont brutes, sans la moindre trace d'enduit ${ }^{22}$. Une des cavités (silo 4) (fig. 2) s'est avérée comblée avec un matériau de remplissage sombre qui a livré des éléments datables du $\mathrm{X}^{\mathrm{e}}$ siècle. Il serait intéressant de pouvoir vérifier la possibilité d'une utilisation en tant que puisard, même si la proximité relative des puits d'eau rend cette identification douteuse.

Dans la partie sud de la ville - un secteur qu'on a récemment proposé d'identifier avec une partie de la cité palatiale fatimide al-Khāliṣa, mais dont il n'est pas exclu qu'elle s'inscrive plutôt dans le «Quartier neuf », le Hāarat al-Djadīda, cité par les sources du X $X^{\mathrm{e}}$ siècle - lors de travaux au Palazzo Statella Spaccaforno, ont été mis au jour les restes d'un habitat qui présente deux phases distinctes : la plus ancienne peut être datée du milieu du $\mathrm{X}^{\mathrm{e}}$ siècle, et la plus récente d'entre la fin du $\mathrm{X}^{\mathrm{e}}$ et le début du $\mathrm{XI}^{\mathrm{e}}$ siècle $^{23}$. Les habitations, dont on conserve d'amples pans, sans pour autant pouvoir reconstituer un plan complet, répondent à trois types qui présentent tous une

20. Je dois cette information à l'amabilité de Lucia Arcifa que je remercie. Sur les recherches à Castello san Pietro, $c f$. L. ArCifA, C. A. Di Stefano, M. H. DE Floris et J.M. PESEZ, « Lo scavo archeologico di Castello S. Pietro a Palermo », B.C.A. Bollettino d'informazione trimestrale per la divulgazione dell'attività degli organi dell'Amministrazione dei Beni culturali e ambientali della Regione Siciliana, a cura dell'Accademia Nazionale di Scienze Lettere e Arti di Palermo, 6-8 (1985-1987), n. 2, p. 30-41 ; ID., « Palerme, quartier Castello San Pietro », Mélanges de l'École française de Rome. Moyen Âge, 101 (1989), 1, p. 332-350 ; L. ARCIFA et A. BAGNERA, « Lo scavo di Castello-San Pietro (Palermo) : una riconsiderazione dei contesti ceramici della prima età islamica », dans A. NEF et F. ARDIZZONE éd., Les Dynamiques..., p. 165-195.

21. E. LESNES, « La recente ricerca archeologica nel Museo », Quaderni del Museo Archeologico Regionale «Antonino Salinas », 3 (1997), p. 17-61.

22. P. TODARO, « La scoperta di alcuni "butti" negli strati calcarenitici nel sottosuolo del Museo "Antonio Salinas" di Palermo », Quaderni del Museo Archeologico Regionale «Antonino Salinas », 3 (1997), p. 63-69 (p. 68).

23. F. SPATAFORA et E. CANZONIERI, « Al-Khālișa ... ». 


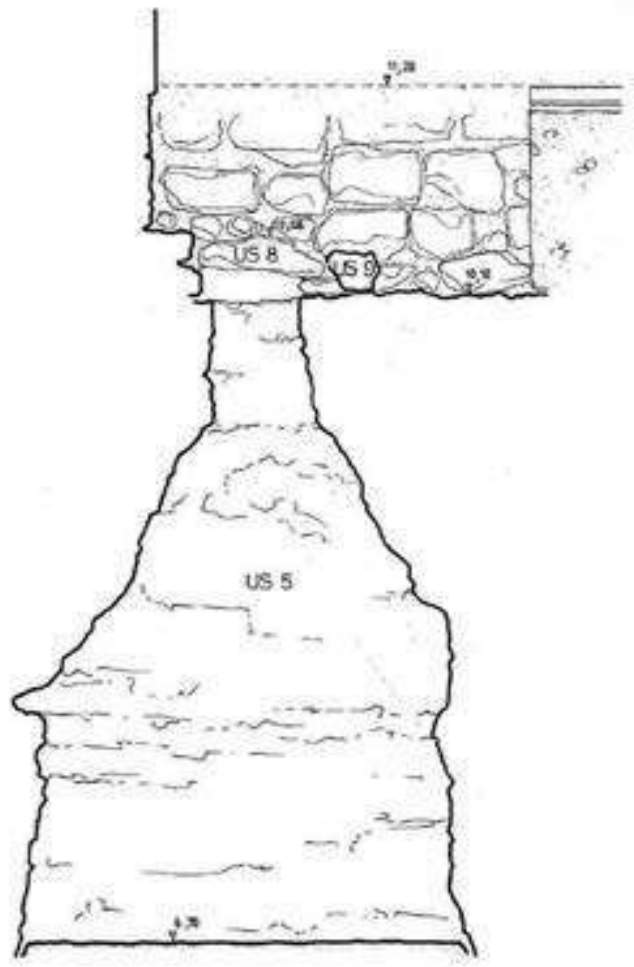

Fig. 2. Musée archéologique régional «Antonio Salinas », silo 4 (D’après E. LESNES, 1997)

cour, séparée de la rue par un mur et dotée d'un puits qui fut, dans certains cas, transformé en dépotoir.

Ces maisons sembleraient dénuées de petits espaces utilisables comme latrines, ou fosses identifiables comme des décharges liées à une latrine située au-dessus. Elles sont distribuées selon deux axes de rues, avec une orientation sud-ouest/nord-est et une légère pente vers la mer ; elles présentent des canalisations couvertes de dalles, utilisées pour la récupération des eaux de pluie ${ }^{24}$. Reste à comprendre comment était résolue la question hygiénique dans chacune de ces maisons, étant donné que ce secteur apparaît caractérisé par un tissu dense, sans espaces vides ni destinés à des jardins. On note enfin que, pour l'heure, dans le panorama morphologique des céramiques découvertes à Palerme et datées de l'époque islamique, aucun récipient n'a été identifié comme vase de nuit ou comme vase lié à l'hygiène personnelle.

Pour synthétiser, on peut dire que la documentation archéologique est encore extrêmement lacunaire et, qu'en outre, elle ne concerne pas les

24. Ibid., p. 238. 
secteurs où se concentraient probablement les résidences des élites, c'està-dire la zone correspondant au noyau antique, Balarm, ou la zone palatiale de la Khālișa. Les rares indices de la présence de latrines - données signalées plus haut à propos d'Hāarat al-Ṣaqāliba - sont des cavités peut-être identifiables comme des puits noirs, mais il semblerait aussi - si l'on s'en tient aux résultats publiés - que dans certaines zones urbaines, comme en rendent compte les trouvailles du palais Statella Spaccaforno, les maisons ne disposaient d'aucune structure destinée à l'hygiène.

Cette donnée, si elle était confirmée, pourrait être un indicateur important des conditions hygiéniques urbaines, et, par extension, de la qualité de vie dans la ville.

Quoi qu'il en soit, ces éléments devront être vérifiés en élargissant les enquêtes et en révisant les résultats des fouilles déjà effectuées. La recherche archéologique viendra peut-être confirmer les propos méprisants d'Ibn Hawqal quand il écrivait des Siciliens : « La saleté qui se trouve dans les maisons des habitants ne pourrait pas être comparée à la saleté des Juifs les plus sales [...]. Chez ceux qui appartiennent à une classe sociale supérieure, les poules salissent les sièges et les oiseaux fientent sur les tapis de prière et sur les coussins ${ }^{25}$. »

\section{Latrines royales}

Si pour la Palerme islamique les données nous font défaut, pour l'époque normande nous ne disposons que d'un seul exemple relativement bien conservé et accessible ${ }^{26}$. La qualité des espaces destinés à l'hygiène - ou commoda - dans les édifices des élites, trouve la quintessence de son expression dans un des exemples les plus accomplis de l'architecture palatiale d'époque normande : la Zisa, dont la construction fut commencée par Guillaume $\mathrm{I}^{\mathrm{er}}(1154-1166)$ et achevée par Guillaume II (11661189) (fig. 3). Malheureusement, bien qu'il s'agisse d'une réalisation exceptionnelle $^{27}$, et malgré le fait qu'au moins trois monographies lui ont

25. IBn HawQAL, « Descrizione di Palermo », trad. A. De Simone, dans R. LA DuCA éd., Storia di Palermo II..., p. 127.

26. Un autre exemple très sembable à celui de la Zisa pourrait être la tour Pisane construite par Roger II dans le palais normand de Palerme ; il est malheureusement inaccessible à l'observation car cette tour abrite aujourd'hui les bureaux de la Présidence de l'Assemblée régionale de Sicile. Voir V. ZORIĆ, « Torre Pisana, sede di al-malik Rugâr a Palermo », dans G. Bordi, I. Carlettini, M. L. Fobelli, M. R. Menna, P. PoGliani éd., L'officina dello sguardo. Scritti in onore di Maria Andaloro, I. I luoghi dell'arte, Rome, 2014, p. 97-108 (p. 103, n. 45).

27. Voir les remarques de R. Di LIBERTO, « Norman Palermo : Architecture between the 11th and 12th Century », dans A. NEF éd., A Companion..., Leyde/Boston, 2013, p. 139-194 (p. 161-163). 

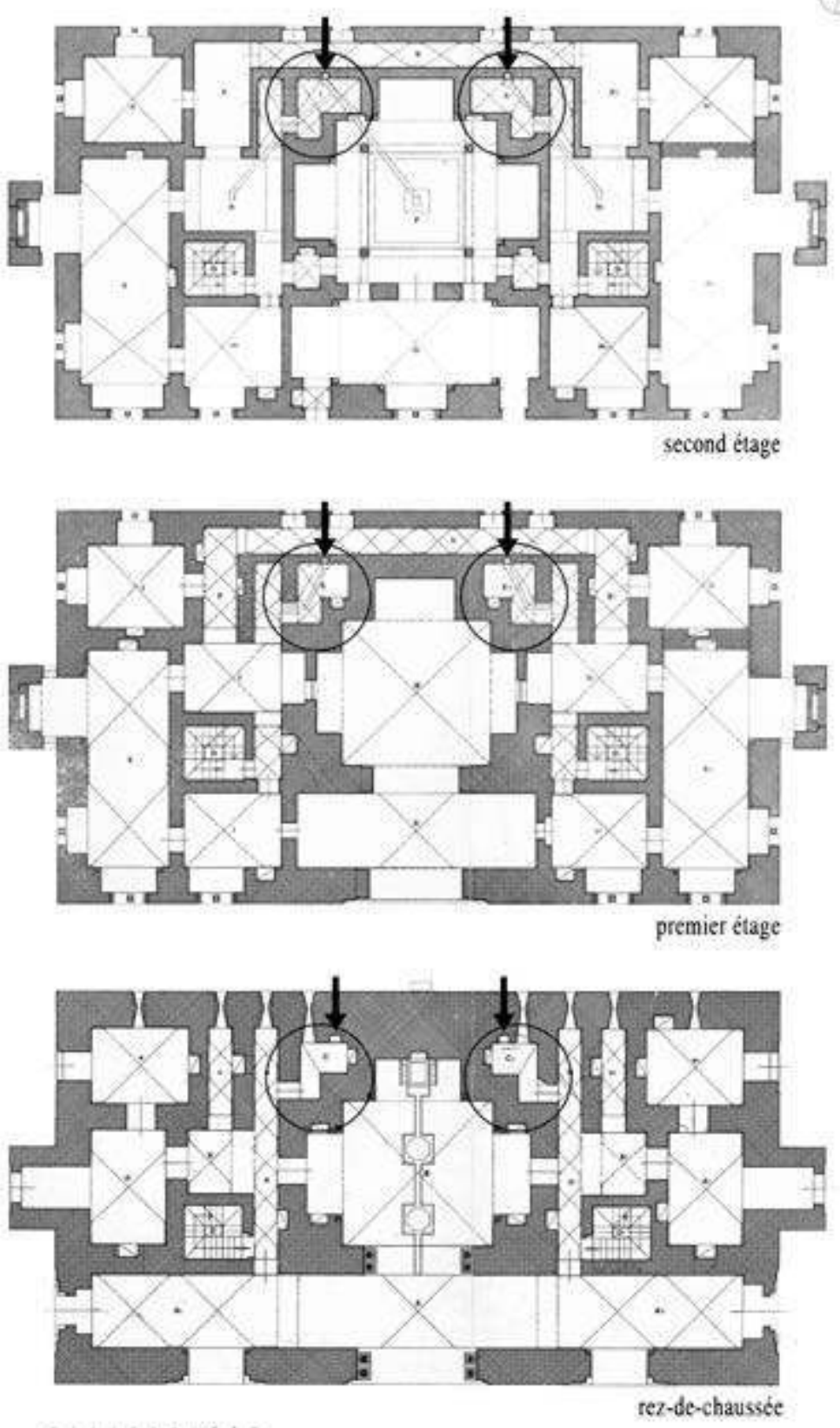

Fig. 3. Palais de la Zisa (Palerme)

Plan des trois étages, avec indication de l'emplacement des latrines et des conduites d'évacuation. (D'après G. BELLAFIORE, 1978 ; repris par Margherita Accascina.) 
été consacrées ${ }^{28}$, la Zisa reste fondamentalement peu étudiée ${ }^{29}$. Du reste, les restaurations effectuées dans les années 1970 ont compromis en partie les possibilités actuelles d'enquêter sur l'édifice, si bien qu'une étude des structures devrait partir d'une révision du matériel documentaire relatif au chantier de restauration.

La Zisa disposait d'un système sophistiqué et raffiné d'équipements sanitaires et de vasques. Malgré les transformations et les adaptations dues à l'utilisation de cet édifice comme demeure des Sandoval (XVII ${ }^{\mathrm{e}}$ siècle), et après l'écroulement de l'aile nord en 1971 et les restaurations consécutives, il reste des indices nets de ce système, mais qui ne permettent pas une reconstruction complète.

D'après les restes encore visibles, et les observations de G. Spatrisano, G. Caronia et G. Bellafiore, qui ont pu avoir une vision directe des structures durant les travaux de restauration, on peut émettre l'hypothèse selon laquelle le système de latrines et d'évacuation des eaux de la Zisa s'articulait de la façon suivante : chacun des trois étages était doté de deux latrines percées dans deux petites pièces coudées situées sur les côtés du corps central ${ }^{30}$. Ces espaces faisaient l'objet d'une bonne isolation : ils étaient fermés par une petite porte et précédés d'un couloir ${ }^{31}$.

Chacun d'eux était aéré grâce à une ou plusieurs fenêtres hautes, et présentait une niche creusée dans un des murs (au rez-de-chaussée, dans le mur sud de la latrine nord, et dans le mur nord de la latrine sud ; aux premier et second étages, dans le mur est). Les niches conservées s'élèvent de $0,72 \mathrm{~m}$ à $1 \mathrm{~m}$ au-dessus du sol, et elles mesurent $0,48 / 49 \mathrm{~m}$ de profondeur, entre 1,40 et $1,10 \mathrm{~m}$ de hauteur et entre 0,68 et $0,80 \mathrm{~m}$ de largeur. Il est probable qu'elles étaient destinées à accueillir un récipient pour l'eau ${ }^{32}$. Deux niches mieux conservées que les autres présentent un encadrement, peut-être la trace d'une corniche en matériau noble, selon un

28. G. Bellafiore, La Zisa di Palermo, Palerme, 1978 ; G. CARonia, La Zisa di Palermo. Storia e restauro, Bari, 1982 ; U. STAACKE, La Zisa : un palazzo normanno a Palermo. La cultura musulmana negli edifici dei re, Palerme, 1991.

29. Sont encore ouvertes certaines questions soulevées par G. Spatrisano et U. Scerrato : G. SpATRISANO, La Zisa ... ; U. SCERRATO, « Arte islamica in Italia », dans F. GABrieli et U. SCERRATO éd., Gli arabi in Italia, Milan, 1979, p. 317-321.

30. Rez-de-chaussée : $2,20 \times 1,50$; premier étage : 2,50 x 1,70 m ; second étage : $3,10 \times$ $1,70 \mathrm{~m}$ (dimensions approximatives).

31. Dans certains édifices de Malaga, mais aussi à l'Alhambra de Grenade, les latrines ont une structure coudée pour mieux les isoler du reste de la maison (L. TORRES BALBÁs, «Letrinas...», p. 225).

32. Des niches s'ouvraient dans les latrines retrouvées sous le couvent de San Francisco à l'Alhambra, et dans les latrines almoravides de la Qubbat Barūdiyyin de Marrakech : selon L. Torres Balbás, il y avait à l'intérieur une vasque pour l'eau (L. TORRES BALBÁs, «Letrinas... », p. 231-232). Une niche se trouve aussi dans les latrines de Castel Maniace à Syracuse, et Vladimir Zorić suggérait qu'elle servait à poser « les récipients avec l'eau, les serviettes et tout le nécessaire pour la propreté et l'hygiène personnelle » : V. ZORIĆ, 
modèle attesté par exemple à Madīnat al-Zahrā', où il est fait recours à des arcs de marbre ${ }^{33}$. Les trois latrines nord et les trois latrines sud sont superposées, même si leurs dimensions ne sont pas les mêmes puisque, lorsqu' on passe du rez-de-chaussée aux niveaux supérieurs, l'épaisseur des murs diminue progressivement, proportionnellement à l'augmentation de la surface interne des pièces.

Dans le mur qui délimite les latrines à l'ouest ont été aménagés deux conduits maçonnés de section quadrangulaire permettant, l'un, l'évacuation des latrines nord, et l'autre, celle des latrines sud. Leur largeur oscille entre 0,35 et 0,40 $\mathrm{m}^{34}$. Elles partent du sol des latrines du second étage et courent jusqu'à un égout placé à la base du mur ouest de l'édifice. Ce conduit, mis au jour lors des fouilles effectuées en $1973^{35}$, consiste en une galerie souterraine de 1,20 m de large et de 1,60 m environ de hauteur, pour partie creusée dans la roche et pour partie construite en pierre de taille de petites dimensions, et couverte d'une petite voûte « peut-être à cintre légèrement brisé $[\ldots]$ et dont le sol est recouvert d'une épaisse couche de béton hydraulique ${ }^{36} »$.

Au moment de la restauration, les conduits contenaient des tubes de céramique que l'on a alors enlevés, de telle sorte qu'il n'est pas possible de déterminer s'ils étaient le résultat d'une intervention postérieure à la construction normande. Un logement pour un tuyau, creusé dans l'épaisseur du mur, très semblable à ceux de la Zisa, est attesté dans la latrine de l'habitation 12 de Madīnat al-Zahrā' ${ }^{37}$. À l'intérieur des latrines du palais palermitain, on ne perçoit pas de traces claires de la cuvette, ou de la liaison entre celle-ci et l'évacuation. On peut toutefois présumer que, dans chaque latrine, la cuvette était appuyée sur le mur ouest, en liaison avec cette dernière.

En outre, les latrines étaient reliées à des conduites qui servaient à évacuer l'eau provenant de certaines vasques placées au second étage, et peut-être aussi au premier $^{38}$. Au second étage du palais se trouvaient trois

« Castello Maniace. L'igiene e gli impianti tecnico-sanitari nell'età sveva », Archivio Storico Siracusano, serie IV, 65 (2010), vol. II, p. 107-192 (p. 136-141).

33. A. Vallejo Triano, La ciudad califal de Madinnat al-Zahrā’. Arqueología de su excavación, Cordoue, 2010, p. 244-245.

34. La largeur est la seule dimension mesurable.

35. V. TUSA, « Sull'archeologia medioevale (con accenni agli scavi eseguiti allo Steri e alla Zisa) », dans Atti del Colloquio internazionale di Archeologia Medievale, Palerme/Erice, 20-22 settembre 1974, Palerme, 1976, p. 104-109 (p. 107).

36. G. SPATRISANO, La Zisa ..., p. 31-32, qui affirme que cela servait à transporter les eaux de pluie provenant des terrasses et les eaux noires des lieux d'hygiène, comme cela apparaît clairement d'après les canalisations d'évacuation intérieures.

37. A. Vallejo Triano, La ciudad..., p. 233, fig. 186 p. 239.

38. À l'Alhambra aussi, les évacuations des latrines recevaient d'abord l'eau des fontaines (L. TORRES BALBÁS, « Letrinas... », p. 223). 
espaces découverts, probablement dotés de bassins (seul celui de l'espace central est conservé), qui recueillaient l'eau de pluie, mais qui étaient sans doute aussi remplis de manière artificielle.

Ces vasques pouvaient être vidées grâce à des canalisations toujours visibles, qui dirigeaient les eaux vers l'évacuation des latrines (fig. 4). Au premier étage, une autre canalisation en pente vers l'écoulement est visible à l'intérieur de la latrine sud ; elle pourrait indiquer soit que, dans un des espaces situé face à elle, était placée une vasque dont le contenu pouvait s'écouler par ce conduit, soit que le projet d'origine du palais ne prévoyait que deux étages.

Les latrines étaient donc reliées à un système de circulation des eaux pures qui, même si elles n'étaient pas courantes, garantissaient un nettoyage périodique. Les déjections étaient ensuite éliminées par un égout situé à l'extérieur.

Pour résumer, les latrines ont été intégrées dans un système de vasques et de canalisations plus complexe et articulé qu'il ne nous apparaît actuellement. Nous ne savons pas, en particulier, s'il y avait aussi un système pour élever l'eau aux étages. De même, la question de savoir si « l'aqueduc sur arcs brisés ? ${ }^{39}$ » visible à l'ouest de l'édifice faisait partie du complexe n'est pas résolue.

La Zisa est un édifice qui trouve ses modèles dans le monde islamique $^{40}$. Ses latrines, avec leur situation à l'intérieur du bâtiment, leur configuration de réduit isolé, avec une entrée coudée, dotées d'une niche et d'un dispositif aussi simple qu'efficace pour l'évacuation, trouvent leurs parallèles les plus étroits dans la sphère de l'architecture palatiale et domestique islamique. Par ailleurs le commanditaire de ce système est un souverain normand et, dans ce nouveau contexte, les six latrines du palais sont des commoditates et contribuent à faire de la Zisa de Guillaume $\mathrm{I}^{\mathrm{er}}$ un édifice « construit admirablement, de la façon la plus confortable et la plus soignée, et qui semblait dépasser toutes les œuvres de son père ${ }^{41} \gg$.

\section{Les latrines dans les résidences castrales de la Sicile occidentale}

Le lien entre lieux destinés à l'hygiène et architecture de prestige est confirmé par la découverte de latrines dans les résidences castrales qui ont fait l'objet d'enquêtes archéologiques.

39. G. SPATRISANO, La Zisa ..., p. 32.

40. U. SCERRATO, «Arte islamica... », p. 308-321 ; R. Di LIBERTO, «Architecture... », p. $162-163$.

41. "Quod commodius ac diligentiuscompositum, videretur universis patris operibus preminere » : Ugo FALCANDO, Historia o Liber de Regno Sicilie, éd. G. B. SiRAguSA, Rome, 1897 (Fonti per la Storia d'Italia 22), p. 87. 


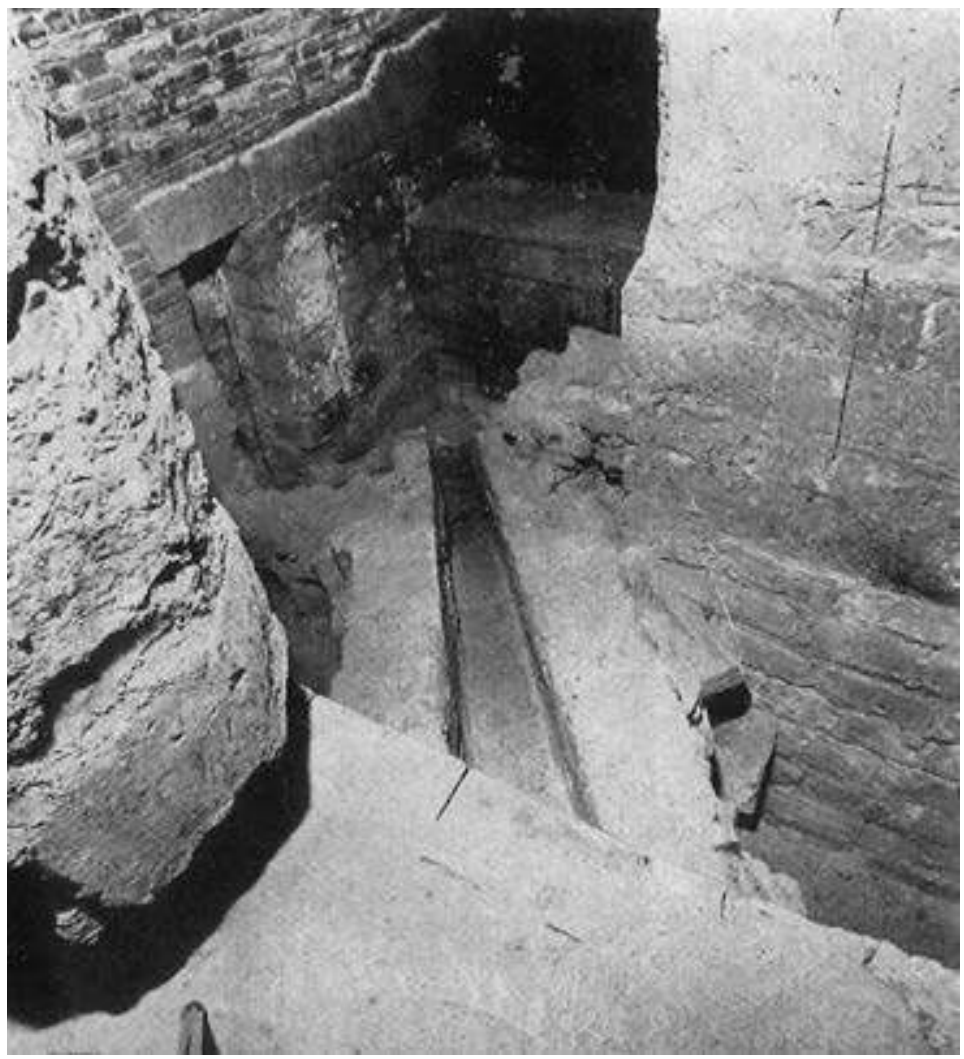

Fig. 4. Palais de la Zisa (Palerme)

Latrine du deuxième étage : au premier plan la canalisation d'eau propre débouchant dans la conduite d'évacuation verticale que l'on devine au second plan.

(D’après G. BELLAFIORE, 1978.)

À Entella, où la population islamique se rassembla lors de sa résistance extrême à Frédéric II, on a mis au jour un ample palais fortifié doté de hammām. Dans l'édifice inférieur attenant au palais, un espace de petites dimensions (pièce 14) conservé sur environ $1 \mathrm{~m} \times 1 \mathrm{~m}$ et immédiatement adjacent à l'entrée de la cour occidentale, a été interprété comme une hypothétique latrine, notamment à cause de la présence d'une grande plaque posée de chant dans l'angle ouest ${ }^{42}$.

42. A. CORRETTI, «1. Entella. Area del palazzo fortificato medievale. Scavo nell'edificio inferiore (SAS $1 ; 2007-08) »$, Annali della Scuola Normale Superiore di Pisa. Classe di Lettere e Filosofia, serie 5, 2/2 Supplemento (2010), p. 53-70 (p. 64). Sur le palais fortifié et sur la possibilité de le mettre en relation avec des figures éminentes de la communauté musulmane, $c f$. A. CORRETTI, « Il palazzo fortificato di Entella », MEFRM, 110 (1998), 2, p. 591-606. 
Des latrines sont attestées également au château de Calathamet ${ }^{43}$, où elles prenaient place dans un petit espace rectangulaire doté d'un canal d'écoulement, mais sans lien direct avec la partie résidentielle du château.

À Ségeste, la latrine fait partie de la première installation du complexe castral, daté des débuts du XIII ${ }^{\mathrm{e}}$ siècle. D'après la reconstitution d'Alessandra Molinari, la latrine se trouvait dans une zone adjacente au corps principal et résidentiel du château, où avait été creusé un petit espace quadrangulaire. À l'intérieur de celui-ci se trouvaient deux murets parallèles hauts d'environ 40 ou $50 \mathrm{~cm}$. On ignore si ces murets étaient ou non couverts d'une plaque. Relié à eux, en tout cas, on trouvait un canal d'écoulement $(30 \mathrm{~cm}$ de hauteur et $30 \mathrm{~cm}$ de largeur), qui charriait les déjections hors de cet espace ${ }^{44}$.

\section{Latrines impériales}

Le raffinement des latrines des châteaux fédériciens de Sicile, qui ne fut pas réservé à Castel del Monte dans les Pouilles, les a fait mettre en relation avec des exemples de latrines de l'architecture palatiale islamique ${ }^{45}$.

Vladimir Zorić a récemment consacré un article monographique aux installations hygiénico-sanitaires de Castel Maniace, à Syracuse, article particulièrement attentif aux aspects tant architecturaux que technologiques ${ }^{46}$. Cette étude relève la présence d'équipements d'hygiène dans les châteaux de Sicile destinés à accueillir Frédéric II - Castel Maniace, le château d'Augusta, le château Ursino à Catane -, mais aussi dans les résidences impériales des Pouilles et de Basilicate à partir de Castel del Monte. En ce qui concerne les équipements sanitaires de Castel Maniace, ils étaient à la hauteur de l'architecture qui les abritait.

Il s'agissait de pièces retirées, auxquelles on accédait en traversant un vestibule, dotées de portes, à l'isolation facile, avec un siège individuel pourvu d'un tuyau d'évacuation qui menait à un niveau inférieur (fig. 5). Ce dernier était à son tour relié à la mer par une galerie, de sorte que le fond était lavé par l'eau de mer. En affirmant que « cet aspect de la manière d'habiter et de vivre, même s'il était indubitablement réservé à la classe dominante, semble avoir caractérisé en particulier le royaume du sud du

43. E. Lesnes et J.-M. Poisson, Calathamet. Archéologie et histoire d'un château normand en Sicile, Palerme, 2013, p. 134-136.

44. A. Molinari, Segesta II. Il castello e la moschea (scavi 1989-1995), Palerme, 1997, p. 73.

45. D. SACK, « Castel del Monte e l'Oriente », dans M. S. CALÒ MARIANI et R. CASSANO éd., Federico II. Immagine e potere, Venise, 1995, p. 295-303 (p. 296).

46. V. ZORIĆ, « Castello Maniace... », p. 107-192. 
grand empereur ${ }^{47} »$, et que peut-être les systèmes d'évacuation des latrines de Castel Maniace sont l'œuvre d'esclaves « sarrasins ${ }^{48}$ », Vladimir Zorić suggère de manière voilée que ces structures reflètent un modèle, un style de vie, lié au monde islamique ou, comme il l'écrit, « oriental ${ }^{49} »$.

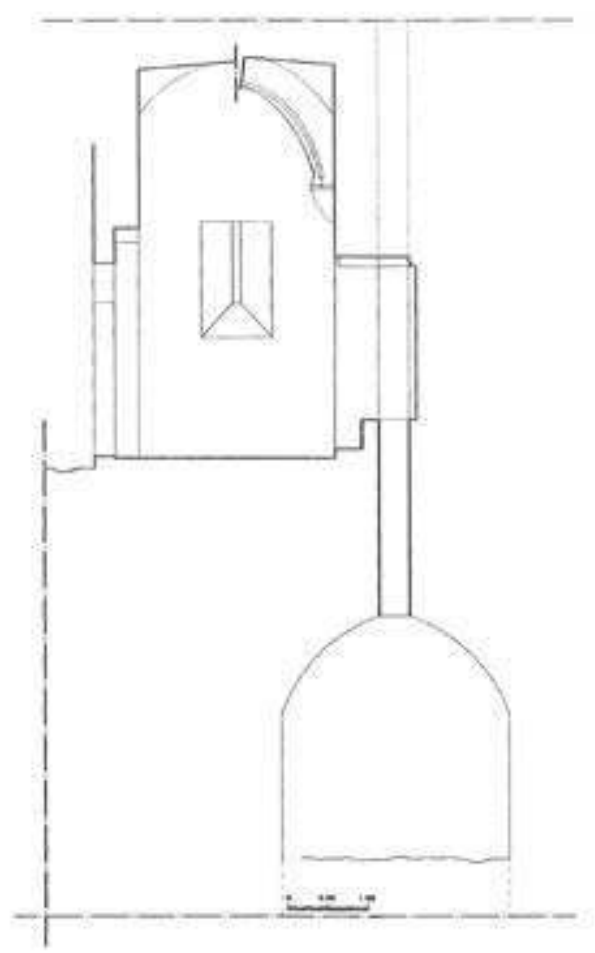

Fig. 5. Castel Maniace (Syracuse)

Coupe verticale de la latrine (garderobe) méridionale.(D’après V. Zorić, 2010.)

La pauvreté des données disponibles sur la Palerme islamique permet seulement de poser des questions en vue d'études futures et de souligner à nouveau l'importance d'un thème de recherche qui est tout sauf secondaire. L'absence, ou la rareté d'attestation de latrines et, plus généralement, de structures dédiées à l'hygiène, si elle est confirmée, construit l'image d'une cité dotée d'amples zones caractérisées par un tissu dense mais économiquement pauvre.

47. Ibid., p 184.

48. Ibid., p. 183, n. 9 .

49. Ibid., p. 192. 
Toutefois, la mention d'un puits perdu, certes dans un document d'époque normande relatif à Catane, et la confrontation avec ce que l'on sait d'al-Andalus à la même époque, invitent à penser que l'on se heurte, dans le cas de Palerme, à un problème d'identification, les traces archéologiques des structures destinées à l'hygiène n'étant peut-être pas reconnues comme telles. Par ailleurs, les données issues des sources écrites et matérielles amènent à émettre l'hypothèse selon laquelle le tissu urbain de Palerme était dense, mais discontinu et hétérogène. C'était une ville à la structure complexe avec des quartiers où se concentraient les « demeures des grands marchands » et celles des personnalités liées à la cour émirale, des zones dans lesquelles prévalaient des structures artisanales, et d'autres dont il est difficile pour le moment de définir les caractères propres ${ }^{50}$. Dans ce contexte, la présence d'installations hygiéniques à l'intérieur des maisons devient un indicateur important de la qualité de l'architecture domestique et, par conséquent, un instrument utile pour reconstituer la topographie urbaine ainsi que les caractéristiques de la ville elle-même.

L'unique exemple bien documenté de latrines à l'époque normande a une telle dimension d'exception qu'on ne peut le considérer comme représentatif des installations sanitaires présentes dans l'ensemble de l'architecture domestique de la Palerme islamique et normande. La Zisa, ce « paradis terrestre qui s'ouvre aux regards », selon l'inscription qui orne le portail d'accès à la salle centrale du rez-de-chaussée, et qui comptait six latrines confortables, s'inscrit dans la koinè méditerranéenne de la représentation de la souveraineté au $\mathrm{XII}^{\mathrm{e}}$ siècle. C'est l'expression d'une cour en contact avec celles du Caire et de Byzance et qui, dans la seconde moitié du XII ${ }^{e}$ siècle, accueille aussi les Hammoudides et les exilés profatimides d'Égypte ${ }^{51}$. Son architecture est le fruit d'un projet organique, incluant également des installations hydrauliques et de complexes systèmes d'aération. Pour ces six latrines raffinées, elle adopte un système d'évacuation élaboré, constitué de tuyaux de décharge reliés à un égout.

Tout aussi exceptionnelles sont les commodités d'époque fédéricienne, qui apparaissent dans des architectures représentatives d'une idéologie complexe du pouvoir impérial, dans un contexte ayant subi aussi de profondes mutations par rapport à celui du royaume normand. La comparaison avec les latrines des architectures castrales, situées à l'intérieur de petits espaces et dotées de simples conduits d'écoulement vers l'extérieur, permet de prendre la mesure de leur singularité. En même temps, les rares exemples de latrines castrales semblent indiquer que les résidences des élites, chrétiennes et peut-être - dans le cas d'Entella - musulmanes,

50. Sur l'organisation de la ville et de ses quartiers, voir A. NEF, «Croissance urbaine...» .

51. A. NEF, Conquérir et gouverner la Sicile islamique aux XI et XII siècles, Rome, 2011 (Bibliothèque des Écoles Françaises d'Athènes et de Rome 346), p. 619-620. 
prévoyaient la présence de lieux réservés à l'hygiène, même si elles les rejetaient à l'extérieur du corps principal de la demeure.

L'association entre latrines et résidences des élites est un trait qui distinguera aussi la ville de Palerme au bas Moyen Âge, pour laquelle les sources attestent une ample gamme de solutions en fonction des possibilités des commanditaires : de petits lieux d'hygiène annexés aux chambres dans les bâtiments de prestige, des latrines creusées dans les cours, des espaces non construits où les habitants résidant autour d'une même cour (cortilis domorum) avaient l'habitude de ménager leur lieu d'aisance ${ }^{52}$ et, pour finir, les simples vases de nuit, parfois associés à de confortables sièges de bois, les « segie de lignuper fari axiu », qui apparaissent dans les inventaires testamentaires des membres des élites ${ }^{53}$.

Il reste que rien n'indique que le type d'installation aulique que nous avons évoqué, qui renvoie à des types de construction bien connues dans le monde islamique médiéval, ait été introduit à Palerme à l'époque normande et pas à une période antérieure, ni que les préoccupations hygiéniques (prenant la forme de l'aménagement de latrines) aient été cantonnées, à l'époque islamique, exclusivement aux demeures des élites palermitaines. Ces attestations, pour l'instant limitées spatialement et socialement, laissent donc ouvertes ces deux interrogations et invitent les archéologues à approfondir l'enquête sur le sujet.

Texte traduit de l'italien par Laurence Moulinier-Brogi

Elena Pezzini - Museo Archeologico Regionale di Palermo "A. Salinas »

\section{Les latrines dans l'architecture domestique et palatiale de Palerme, un marqueur social ?}

Le peu d'informations disponibles sur l'architecture domestique de Palerme islamique ne permet pas de comprendre si le nombre très réduit d'attestations de latrines est dû à une véritable absence de celles-ci ou à la difficulté à en reconnaître les vestiges. Quelques indices semblent confirmer toutefois leur existence, montrant de plus qu'elles étaient associées à des fosses. Quant aux sources textuelles, elles suggèrent que le tissu urbain n'était pas homogène et que les résidences des élites, plus sûrement dotées d'installations sanitaires, étaient concentrées dans des quartiers que nous connaissons peu, car les données archéologiques publiées sont rares et leur interprétation reste difficile. Pour l'époque normande, en revanche, le palais

52. « solebant facere privatam sive clohacam aut necessarium»: E. PEZZINI, « Lo smaltimento... », p. 17-19.

53. G. BResC-BAutier, « Pour compléter les données de l'archéologie : le rôle du bois dans la maison sicilienne (1350-1450) », dans Atti del Colloquio Internazionale di Archeologia Medievale (Palerme/Erice, 20-22 septembre 1974), Palerme, 1976, II, p. 435464 (p. 460, n. 72) ; G. BRESC-BAUTIER et H. BRESC, Une maison de mots. Inventaires de maisons, de boutiques, d'ateliers et de châteaux de Sicile (XIII-XVe siècles), Palerme, 2014 (Associazione no profit Mediterranea), I, p. 105-106. 
de la Zisa conserve un remarquable ensemble de latrines qui doivent être rapprochées de celles connues dans l'architecture palatiale islamique. Mais le caractère exceptionnel de ce palais, construit dans la seconde moitié du $\mathrm{XII}$ siècle, interdit de généraliser ces observations à l'architecture domestique palermitaine antérieure ou contemporaine. Le lien entre lieux d'aisance et résidences des élites est réaffirmé par la redécouverte de latrines tant au château de Ségeste que dans l'édifice inférieur du palais fortifié d'Entella - ville que les sources islamiques considèrent comme le siège du prétendu " émirat des Montagnes ". Des études récentes, enfin, ont mis en relation les latrines extrêmement raffinées des châteaux fédériciens (Castel Maniace à Syracuse, Castello di Augusta) avec certains exemples de l'architecture résidentielle islamique.

Architecture palatiale normande - latrines - Palerme islamique - puits perdus

\section{Latrines in the Domestic and Palatial Architecture of Palermo}

The little information that we have on domestic architecture in Islamic Palermo makes it impossible to understand whether the reduced number of latrines is due to a problem of recognition of the archaeological datum or to the fact that there really were few such structures. Some evidences allow us to affirm that there were latrines and that they were connected to cesspits, while the sources indicate that Palermo was a city with a heterogeneous fabric in which the residences of the elites, presumably fitted out with sanitation, were concentrated in some districts that are little known because published archaeological data are few and difficult to interpret. By contrast, for the Norman age in the Zisa palace an exceptional system of latrines is preserved that is comparable to the latrines in Islamic palatial architecture and nevertheless, because of its exceptional character, cannot be considered indicative of the type of sanitation present in domestic architecture in Islamic and Norman Palermo. The connection between latrines and residences of elites is confirmed by the recovery of latrines both in Segesta Castle and in the lower building of the fortified palace at Entella, a city that Islamic sources identify as the center of the so-called "emirate of the Mountains". Lastly, recent studies have connected to examples attested in Islamic residential architecture the very refined latrines of the castles of Frederick II (Castel Maniace in Syracuse, Augusta Castle).

Cesspits - Islamic Palermo - Latrines - Norman Sicilian Palaces 